\title{
3D numerical simulations of magnetic field evolution in barred galaxies and in spiral galaxies under influence of tidal forces
}

\author{
Katarzyna Otmianowska-Mazur ${ }^{1}$, Katarzyna Kulpa-Dybeł ${ }^{1}$, Barbara \\ Kulesza-Żydzik ${ }^{1}$, Hubert Siejkowski ${ }^{1}$ and Grzegorz Kowal ${ }^{1,2}$ \\ ${ }^{1}$ Astronomical Observatory, Jagiellonian University, \\ ul Orla 171, 30-244, Kraków, Poland \\ email: otmian@oa.uj.edu.pl \\ ${ }^{2}$ Instituto de Astronomia, Geofisica e Ciencias Atmosfericas, Universidade de Sao Paulo, Rua \\ do Matao 1226, EP 05508-900, Sao Paulo, Brazil \\ email: kowal@astro.iag.usp.br
}

\begin{abstract}
We present the results of the three-dimensional, fully non-linear MHD simulations of the large-scale magnetic field evolution in a barred galaxy with the back reaction of magnetic field to gas. We also include the process of the cosmic-ray driven dynamo. In addition, we check what physical processes are responsible for the magnetic field evolution in the tidally influenced spiral galaxies. We solve the MHD equations for the gas and magnetic field in a spiral galaxy with gravitationally prescribed bulge, disk and halo which travels along common orbit with the second body. In order to compare our modeling results with the observations we also construct the maps of high-frequency (Faraday rotation-free) polarized radio emission from the simulated magnetic fields. The model accounts for the effects of projection and limited resolution.

We found that the obtained magnetic field configurations are highly similar to the observed maps of the polarized intensity of barred galaxies, because the modeled vectors form coherent structures along the bar and spiral arms. We also found a physical explanation of the problem of inconsistency between the velocity and magnetic fields character present in this type of galaxies. Due to the dynamical influence of the bar, the gas forms spiral waves which go radially outward. Each spiral arm forms the magnetic arm which stays much longer in the disk than the gaseous spiral structure. The modeled total energy of magnetic field and magnetic flux grows exponentially due to the action of the cosmic-ray driven dynamo. We also obtained the polarization maps of tidally influenced spiral galaxies which are similar to observations.
\end{abstract}

Keywords. galaxies: evolution, magnetic fields, ISM: cosmic rays, methods: numerical, MHD

\section{Introduction}

The radio synchrotron observations of disks of nearby galaxies (spiral and barred) indicate that magnetic fields are present in a big number of them (Beck 2009). Moreover, they show that the total strength of the magnetic field is in the range of $10-30 \mu \mathrm{G}$ in the spiral arms. In the interstellar medium (ISM) such strength is dynamically important, because the magnetic field has similar mean energy density to the gas and to cosmic rays. In the spiral arms we mainly observe a random component of the magnetic field, while the maxima of polarized emission distributed along gaseous arms are visible in interarm regions (Beck 2009).

The sample of 20 radio emission maps of barred galaxies (Beck et al. 2002) show that the central region of the galaxy NGC 1365 has the strongest polarized emission. In the outer disk of this galaxy the polarized magnetic arms are well visible between the bar and spiral arms similarly to spiral galaxies. Furthermore, the synchrotron observations 
of barred galaxies indicate the presence of regions located upstream the dust lanes, in which the polarization vectors change quickly their pitch angles what results in creation of depolarization valleys within the telescope beam. The same observations of vanishing polarized intensity are observed near shear shock areas. In several barred galaxies the topology of magnetic field is very similar to NGC 1365 (e.g. NGC 1672, NGC 7552 and NGC 1097- Beck et al 2002). In spiral galaxies the synchrotron observations also reveal the presence of strong polarized halos extending few kiloparsecs from the disks and having the X-shape topology.

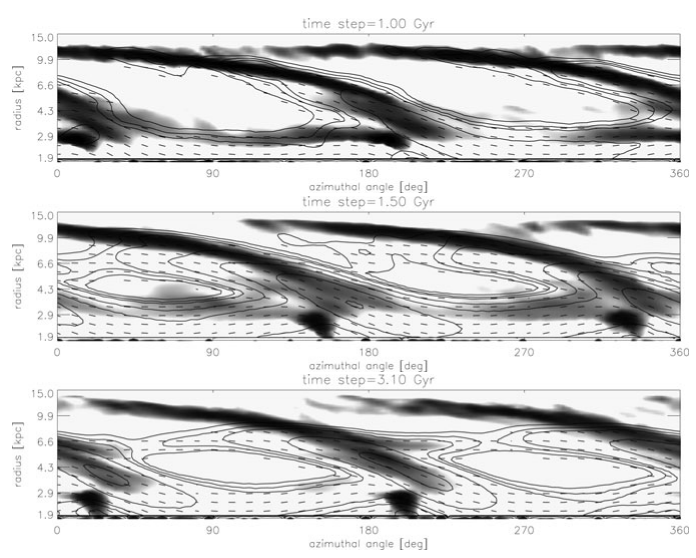

Figure 1. Distribution of polarized intensity and B-vectors superimposed on the gas density in the frame of azimuthal angle in the disk and the logarithm of distance from the center for three time steps: 1.0 Gyr, 1.5 Gyr, 3.1 Gyr. The map has been convolved to the resolution 40".

The development of the magnetic field in barred galaxies was studied numerically by many authors (e.g. Otmianowska-Mazur et al. 2002, Moss et al. 2007, Kulesza-Żydzik et al. 2009 - Paper I, Kulesza-Żydzik et al. 2010 - Paper II), however, the models including the classical dynamo theory in this calculations did not show the promising results (Moss et al. 2007). In opposition to them in our two papers (Paper I \& II) we presented numerical models which are capable to explain the drift of magnetic field arms into the interarm region.

In our simulations, made with the help of Godunov code (Kowal et al. 2009, see also Paper I \& II), we apply the nonaxisymmetric gravitational potential of a bar rotating as a solid body and a galactic disk rotating differentially. The drift of magnetic arms can be explained as follows. In the beginning, due to a faster rotation of the bar than the disk, gas in a differentially rotating disk concentrates in the spiral arms. Due to the shear and compression these gaseous arms induce similar magnetic arms distributed along them. The further rotation of the bar, being in agreement with its pattern speed, produces the new package of spiral arms together with the new magnetic ones. The "old" magnetic arms stay between the spiral arms together with the gas in the disk. Such mechanism could happen even few times during the evolution time of a barred galaxy (see Paper I \& II). In the present 3D MHD numerical simulations of a barred galaxy we additionally include the cosmic-ray driven dynamo process (see Hanasz et al. 2009). We would like to learn how such realistic mechanism as supernova actions in the disk can change the magnetic field evolution in our modeled barred galaxy. Also, in order to check how the process of shifting of the magnetic arms works in spiral galaxies under the influence of tidal forces, we constructed a model of spiral galaxy orbiting around the center of mass where we do not apply cosmic ray driven dynamo process. 


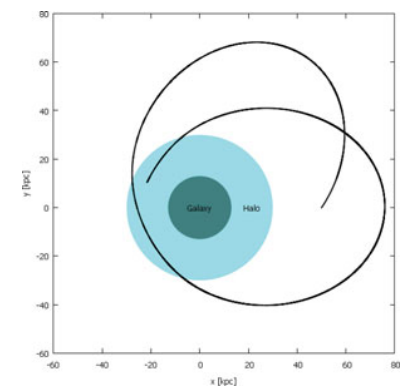

Figure 2. The position of the big galaxy together with the orbit of the small galaxy.
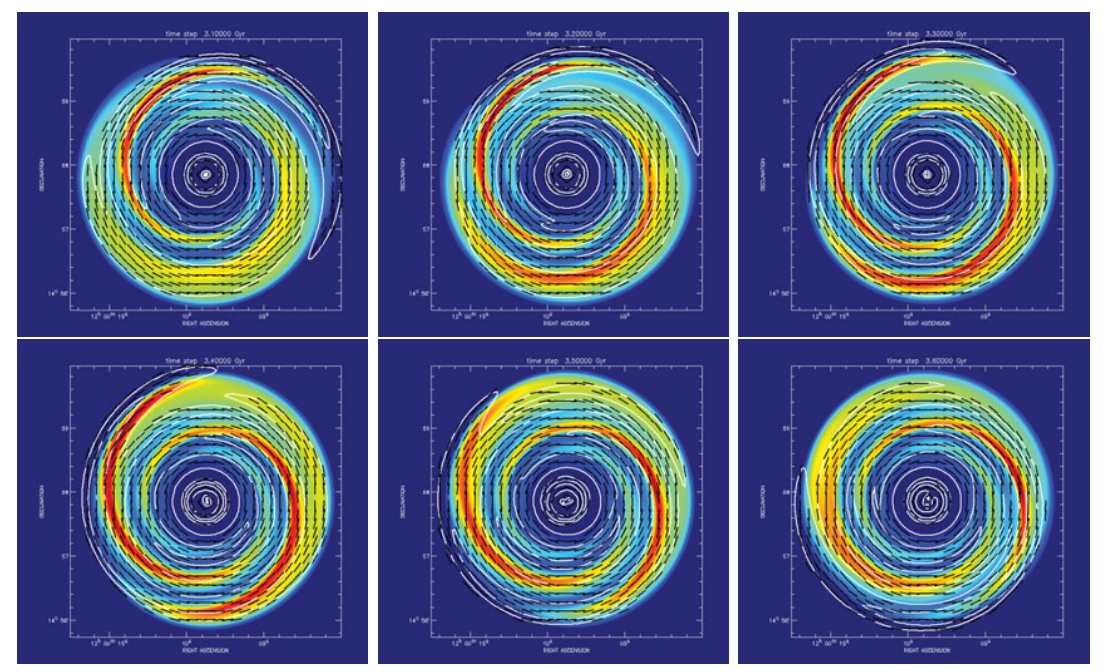

Figure 3. The polarization maps of the big galaxy superimposed onto the density color maps at six time steps 3.1 Gyr 3.2 Gyr, 3.3 Gyr, 3.4 Gyr, 3.5 Gyr, 3.6 Gyr.

\section{Results of magnetic field evolution in barred galaxies with CR dynamo}

First of all, we describe the problem of shifting of magnetic arms into the inter-arm region. The barred galaxy in our model is simulated in the Cartesian grid with the size of $30 \mathrm{kpc} \times 30 \mathrm{kpc} \times 7.5 \mathrm{kpc}$ in $\mathrm{X}, \mathrm{Y}$ and Z direction, respectively (Kulpa-Dybeł et al. this Volume - see also the exponential growth of the magnetic flux). The mass of the stars in the whole galaxy is assumed to be $1.75 \times 10^{11} M_{\odot}$. In addition, we apply the mass of the bar which equals $1.5 \times 10^{10} M_{\odot}$ with the size of $6 \mathrm{kpc} \times 3 \mathrm{kpc} \times 2.5 \mathrm{kpc}$. The bar rotates with the speed of $30 \mathrm{~km} / \mathrm{s}$. Using the simulated magnetic field and cosmic rays distribution we computed the polarization maps which can be compared directly with the polarization maps of the real galaxies.

In Fig. 1 we present our results in a graph showing the distribution of polarized intensity and B-vectors orientation superimposed on the gas density in the frame of azimuthal angle in the disk and the logarithm of distance from the center for three time stages: 1.0 Gyr, 1.5 Gyr, 3.1 Gyr. The map at the first time step (1.0 Gyr) reveals that the gaseous spiral arms just induced the magnetic arms which are exactly distributed along them. The next stage at 1.5 Gyr shows a shift of the magnetic arm from the left side of the gaseous arms to the interarm region. The last graph presents that magnetic field is mainly in the interam regions, however a part of the polarized intensity is also visible 
along the spiral arms in the region close to the center. Such behavior of magnetic arms shows that the process of shifting them to the interarm region is present.

\section{Results of the magnetic field evolution in the spiral galaxies under the tidal force without the CR dynamo}

In order to check how the process of formation of the spiral and magnetic arms looks in spiral galaxies under the influence of tidal motions we prepare a model of the disk galaxy gravitationally disturbed by a second small body.

Fig. 2 reveals the orbit of the small body which starts its movement at the right side of the picture and ends in the halo of the big galaxy presented by disk $\left(8.0 \times 10^{10} \mathrm{M}_{\odot}\right)$ and halo $\left(7.3 \times 10^{11} \mathrm{M}_{\odot}\right)$ as color plots. Initially, the disk rotates differentially with the circular velocity of $200 \mathrm{~km} / \mathrm{s}$. The size of our big modeled galaxy is $30 \mathrm{kpc} \times 30 \mathrm{kpc} \times 7.5 \mathrm{kpc}$ with the resolution of $256 \times 256 \times 64$ in $\mathrm{X}, \mathrm{Y}$ and $\mathrm{Z}$, respectively. In the beginning we assume the hydrostatic equilibrium with $p_{\text {mag }} / p_{\text {gas }}=10^{-6}$ with the axisymmetrical magnetic field component.

Fig. 3 shows six maps of the computed polarization vectors superimposed onto the density maps at six time stages in Gyrs: 3.1, 3.2, 3.3, 3.4, 3.5, 3.6. We see that tidal forces induce the spiral arm very quickly, and the magnetic structures are also formed due to the compression and shear connected with the gas motion. In the first presented snapshot we observe the magnetic arms distributed along the spiral ones, polarized vectors in the interarm region and the region without magnetic field along the second spiral arm. In the second picture (3.2 Gyr) the big region with no magnetic field is present. In the third map (3.3 Gyr) we can see that one magnetic arm could be a bit faster than the gaseous one going into the inter-arm region down and for the next two time steps (3.4 Gyr, 3.5 Gyr) this process continues. In the end at the time 3.6 Gyr we can see only the new magnetic arm distributed along the spiral arm. We can observe here also the magnetic field in the whole disk. In order to demonstrate that the process of shifting of magnetic arms into inter-arm regions exists, although not so strong as in the barred galaxies, we plan to make an analysis of the behavior of the spiral waves together with the magnetic arms.

\section{Conclusions}

In the calculations of magnetic field evolution in barred galaxies, our model reproduces the large-scale structures of magnetic field observed in such galaxies. In the same time our simulations solve the problem of the shift of the magnetic arms to the interarm region. Our second experiment shows that under the influence of a tidal force, the galaxies form magnetic and spiral arms, reproducing the large-scale structures of magnetic field observed in spiral galaxies.

\section{References}

Beck, R. et al. 2002, A\& A, 391, 83

Beck, R. 2009, Astrophys. Space Sci. Trans., 5, 43

Hanasz, M., Wóltański, D., \& Kowalik, K. 2009, ApJ, 706, 155

Kowal, G., Lazarian, A., Vishniac, E. T., \& Otmianowska-Mazur, K. 2009, ApJ, 700, 63

Kulesza-Żydzik, B., Kulpa-Dybeł, K., Otmianowska-Mazur, K., Kowal, G., \& Soida, M. 2009, $A \mathscr{G} A, 498 \mathrm{~L}, 21$

Kulesza-Żydzik, B., Kulpa-Dybeł, K., Otmianowska-Mazur, K., Soida, M., \& Urbanik M. 2010, $A \mathscr{G} A 522,61$

Moss, D., Snodin, A., Englmaier, P., Shukurov, A., Beck, R., \& Sokoloff, D. 2007, A\&\&A 465, 15 